\title{
Cuidadores familiares campesinos: carga de cuidado, tiempo de cuidado y grado de funcionalidad ${ }^{1}$
}

\author{
Vilma Velásquez² \\ Lucero López3 \\ Yenny Barreto 4
}

doi:10.11144/Javeriana.IE16-2.cfCC

Cómo citar: Velásquez V, López L, Barreto Y. Cuidadores familiares campesinos: carga de cuidado, tiempo de cuidado y grado de funcionalidad. Investig Enferm. Imagen Desarr. 2014;16(2):65-80. doi:10.11144/Javeriana.IE16-2.cfcc

1. Artículo de investigación. Recibido: 27 de enero de 2014. Revisado: 4 de febrero de 2014. Aprobado: 23 de mayo de 2014. Trabajo enmarcado en los proyectos del grupo de investigación en Cuidado Cultural de la Salud, con el apoyo financiero de la Facultad de Enfermería de la Universidad Nacional de Colombia, código: 12963, en el marco de la Convocatoria UGI-2010 para financiación de proyectos y publicación de resultados de investigación de los grupos de investigación de la Facultad de Enfermería.

2. Enfermera. MSc. en Enfermería. Profesora asociada, Facultad de Enfermeria, Universidad Nacional de Colombia, sede Bogotá. Investigadora del Grupo de Investigación Cuidado Cultural de la Salud. Correo electrónico: vvelasquez@unal.edu.co

3. Enfermera. MSc en Enfermeria. PhD en Enfermeria. Profesora asociada, Facultad de Enfermería, Universidad Nacional de Colombia, sede Bogotá. Líder del Grupo Investigación en Cuidado Cultural de la Salud. Correo electrónico: allopezdi@unal.edu.co

4. Enfermera, Estudiante de la Maestría en Salud Pública de la Universidad Nacional de Colombia. Joven investigadora de Colciencias. Integrante del Grupo de Investigación Cuidado Cultural de la salud. Correo electrónico: ymbarretoz@unal.edu.co 


\section{Resumen}

Objetivo: Establecer la asociación de la carga del cuidador familiar con el tiempo de cuidado y el grado de funcionalidad de la persona anciana campesina. Materiales y método: Estudio cuantitativo de tipo transversal con alcance correlacional, con una muestra de 50 cuidadores familiares. Fueron aplicados: una encuesta sociodemográfica; escala de Zarit, que mide la carga del cuidador y el índice de Barthel, que mide el grado de dependencia funcional. Resultados: Los cuidadores familiares generalmente son mujeres (76\%); mayores de 60 años (50\%), casados (40\%) y solteros (30\%); con bajo nivel socioeconómico (94\%) y educativo (66\%). No se encontró una asociación significativa entre carga del cuidador y el grado de funcionalidad $(\mathrm{p}=0,249)$, tampoco entre la carga del cuidador $\mathrm{y}$ el tiempo como cuidador $(\mathrm{p}=$ 0,476). Conclusiones: Alta proporción de mujeres campesinas son cuidadoras familiares. El estudio reporta que el tiempo de cuidado y el grado de funcionalidad del anciano no son predictores de carga de cuidado en el cuidador familiar campesino. Se requieren más investigaciones e indagar si hay aspectos culturales que influyen en la experiencia y construcción de significados del cuidado. Se sugiere la necesidad de programas educativos dirigidos a cuidadoras campesinas mediante procesos de autorreconocimiento y autodeterminación de acuerdo con su cultura y capacidades.

Palabras clave: cuidadores; anciano frágil; población rural

\section{Peasant Family Caregivers: Care Burden, Care Time and Level of Functionality}

\section{Abstract}

Objective: To establish the association of the burden of family caregivers with care time and the degree of functionality of the elderly peasant. Materials and methods: quantitative study with cross-sectional correlational range, with a sample of 50 family caregivers. It was applied: one sociodemographic survey; Zarit scale, measuring caregiver burden, and the Barthel Index, which measures the degree of functional dependence. Results: Family caregivers are usually women (76\%); over 60 (50\%), married (40\%) and single (30\%); with low socioeconomic status (94\%) and education (66\%).No significant association between caregiver burden and the degree of functionality ( $p=0.249$ ) was found, nor between caregiver burden and time as caregiver $(\mathrm{p}=0.476)$. Conclusions: A high proportion of rural women are family caregivers. The study reports that caring time and degree of functionality are not predictors of elder care burden on the peasant family caregiver. More research and inquire is needed to determine if there are cultural aspects that influence the experience and meaning construction of care. The need for educational programs for rural carers through processes of self-recognition and self-determination in accordance with their culture and capabilities is suggested.

Keywords: rural caregivers; frail elderly; rural population 


\section{Cuidadores familiares camponeses: carga de cuido, tempo de cuido e grau de funcionalidade}

\section{Resumo}

Objetivo: Estabelecer a associação entre a carga do cuidador familiar e o tempo de cuido, e grau de funcionalidade da pessoa idosa camponesa. Materiais e método: Estudo quantitativo de tipo transversal e escopo correlacional, com amostra de 50 cuidadores familiares. Foram aplicados: inquérito sociodemográfico; escala de Zarit, que mede a carga do cuidador, e índice de Barthel, que mede o grau de dependência funcional. Resultados: Os cuidadores familiares geralmente são mulheres (76\%); maiores que 60 anos (50\%), casados (40\%) e solteiros (30\%); de baixo nivel socioeconómico (94\%) e educativo (66\%). Não foi encontrada associação significativa entre carga do cuidador e grau de funcionalidade ( $\mathrm{p}=0,249)$, nem entre carga do cuidador e tempo como cuidador $(p=0,476)$. Conclusões: Alta proporção de mulheres camponesas são cuidadoras familiares. O estudo reporta que o tempo de cuido e grau de funcionalidade do idoso não são preditores de carga de cuido no cuidador familiar camponês. É preciso mais pesquisa e investigar se existem aspectos culturais que influenciam a experiência e construção de significados do cuido. Sugere-se a necessidade de programas educativos direcionados para cuidadoras camponesas mediante processos de autorreconhecimento e autodeterminação de acordo com sua cultura e capacidades.

Palavras chave: cuidadores; idoso fraco; população rural 


\section{Introducción}

La proporción de personas de 60 y más años está en aumento en el mundo. Se proyecta que en 2050 la proporción de estas personas mayores llegará al $21 \%$. Este fenómeno ha afectado a algunos países y traído consecuencias directas en las relaciones dentro de la familia, los estilos de vida, la solidaridad familiar, el crecimiento económico, el ahorro, los mercados laborales, las pensiones, los impuestos, así como la asistencia prestada por una generación a otra, la salud y la atención médica, la composición y organización de la familia, la vivienda y las migraciones (1-3).

Históricamente, la familia ha desempeñado un papel importante en el cuidado de la persona anciana (4). Por lo general, las personas con alta dependencia funcional para las actividades de la vida diaria (bañarse, comer, desplazarse, etc.) deben ser cuidadas por una persona que, en la gran mayoría de los casos, forma parte del núcleo familiar (5). El familiar que asume las responsabilidades del cuidado de la persona mayor es conocido como cuidador familiar, definido por algunos autores como "la persona, habitualmente un familiar directo, que convive y mantiene la responsabilidad de proveer recursos que un paciente incapaz de auto sustentarse necesita" (6).

Los cuidados en la familia se constituyen en un compromiso moral en situaciones de dependencia funcional y las mujeres tienen más probabilidades de cuidar como consecuencia de una decisión familiar. Los cuidadores de personas con discapacidades más graves se sienten agobiados con mayor frecuencia que quienes cuidan personas con discapacidad moderada. La percepción de agobio y de escasez de tiempo es más intensa entre los cuidadores con empleo que entre los cuidadores sin empleo (7). Varios cuidadores familiares afirman que el cuidado se constituye en una experiencia cotidiana del diario vivir y una fuente de cariño y compensación por lo recibido antes por su familiar. La percepción de carga aumenta cuando la decisión de cuidar no es iniciativa del cuidador (7).

En el mundo, reportes de la literatura indican que el rol como cuidadoras familiares ha sido desempeñado principalmente por mujeres (8-11). El rango de edad oscila entre 23 y 87 años. La relación de parentesco más frecuente es la de hija, con un nivel educativo bajo (12) y llevan más de un año cuidando (13).

Diversos estudios (14-17) sobre los cuidadores de personas con dependencia funcional reportan las consecuencias que trae consigo la tarea de cuidar a un familiar, en especial porque se deja de lado el autocuidado para cuidar del otro. Esta situación y las diversas renuncias desde lo económico y social generan un desbalance entre las demandas y las capacidades de los cuidadores, fenómeno conocido como carga del cuidado (5), expresión ampliamente utilizada para caracterizar las frecuentes tensiones y demandas experimentadas de manera subjetiva y objetiva por los cuidadores.

Por lo anterior, la carga del cuidado se clasifica en dos tipos de acuerdo con los efectos que ocasiona en el estado emocional o sobre la vida cotidiana: la carga subjetiva, definida como "las reacciones emocionales ante la experiencia de cuidar" (1,18-20) o la "manera como los cuidadores se sienten acerca de su papel” (21), y se relaciona con la forma en que se 
percibe la situación, tanto las dificultades como las recompensas (1). La carga objetiva es definida como "la carga del cuidador que implica gran cantidad de energía y tiempo dedicado para la realización de tareas que pueden ser desconocidas, desagradables, e incómodas" (21) o "el grado de perturbaciones o cambios en diversos aspectos del ámbito doméstico y la vida de los cuidadores" (20).

Es importante tener en cuenta que el cuidador familiar no tiene formación en salud, cumple con este papel sin someterse a normas de horario ni de procedimiento (22). Las mujeres experimentan mayores cargas en comparación con los cuidadores hombres $(13,23-25)$, debido a que ellas tienen menores dominios (13) y uso menos eficaz de las estrategias de afrontamiento (26); mientras que los hombres utilizan en su mayoría el enfoque de resolución de problemas (25).

Algunos estudios han investigado si el grado de dependencia en las actividades básicas cotidianas $(A B C)$ se relaciona de forma directa con la carga percibida por el cuidador; se encontró que existe una asociación estadisticamente significativa entre sobrecarga y mayor cantidad de enfermedades crónicas que generan dependencia funcional $(p<0,01)(12,27-29)$; sin embargo, son pocos los estudios con poblaciones rurales (30). Al igual, pocas investigaciones reflejan la asociación entre sobrecarga de cuidador y tiempo de cuidado dedicado al cuidado (31) de adultos mayores con dependencia funcional.

En el Grupo de Investigación Cuidado Cultural de la Salud de la Facultad de Enfermería de la Universidad Nacional de Colombia (GCCS-FE, UNC) ha desarrollado diversos trabajos con poblaciones ancianas en situación de discapacidad y pobreza, con sus familias en contextos rurales y urbanos de algunos lugares del país (32-39). Se encontró que el cuidado familiar a la persona anciana dependiente funcional, tanto en grupos rurales como urbanos en situación de pobreza, responde a valores y creencias de reciprocidad, obligación filial, afecto y deseo de ayudar.

En Cáqueza, Cundinamarca, se han realizado estudios etnográficos y de mediaciones culturales en el ámbito domiciliario con personas ancianas campesinas y sus cuidadores, dada la importancia que representa este grupo social y considerando, como plantea Forero (40), que en Colombia las personas que viven en los campos representan el $31 \%$ de la población total, y la mayor parte de los campesinos son productores familiares agropecuarios. Las comunidades campesinas difieren según sus características culturales, ecológicas, evolución histórica, etc., pero todas se caracterizan por tener relaciones de producción basadas en el trabajo familiar o bajo formas colectivas entre los campesinos. Producen sus alimentos básicos con sistemas ancestrales y escasa dotación de recursos, siendo los focos donde se concentra la extrema pobreza (41). Así, el término campesino puede aplicarse a cualquier persona que se ocupe de la agricultura, la ganadería, la trashumancia, las artesanías relacionadas con la agricultura u otras ocupaciones similares en una zona rural; que trabajan la tierra por sí mismos, y que dependen sobre todo del trabajo en familia y otras formas en pequeña escala de organización del trabajo (42). 
Para el caso del estudio se entiende por cuidadores familiares campesinos a las personas que residen en el área rural del municipio de Cáqueza, son productores familiares agropecuarios, cuidan a un familiar con dependencia funcional y participan del programa del municipio: Atención al Adulto Mayor con Discapacidad.

\section{Materiales y métodos}

La investigación realizada es cuantitativa, de tipo transversal con alcance correlacional. De un total de 200 cuidadores campesinos participantes del programa de Atención al Adulto Mayor con Discapacidad, del municipio de Cáqueza, Cundinamarca, la muestra estuvo conformada por 50 personas que aceptaron voluntariamente participar y contaron con los recursos para desplazarse desde las veredas al casco municipal y respondieron a los siguientes criterios de inclusión: ser cuidador familiar de persona anciana con limitación física o sensorial no cognitiva o mental y dependiente de un cuidador familiar para las actividades de la vida diaria; ser productor familiar agropecuario en la zona rural del municipio de Cáqueza y haber cuidado por un mes o más a la persona anciana inscrita en el programa de Adulto Mayor del municipio y ser mayor de 18 años de edad. Todos los participantes aceptaron voluntariamente participar en el proyecto y firmaron un consentimiento informado que incluía autorización para tomar fotos, grabar y confidencialidad de sus datos personales. Se aseguró la protección de la identidad de los participantes por medio de un código asignado.

El proceso para la recolección de la información consideró los siguientes pasos: a) contacto con la Coordinación de Salud Pública de la Alcaldía de Cáqueza; b) invitación por medio de la emisora La Voz de Cáqueza a los cuidadores familiares participantes del programa de Atención al Adulto Mayor con Discapacidad del municipio de Cáqueza; c) encuentro con los cuidadores y presentación del proyecto; d) elección de participantes y firma del consentimiento informado, y e) aplicación de instrumentos entre noviembre y diciembre de 2011.

\section{Instrumentos}

Fueron aplicados una encuesta sociodemográfica a los cuidadores familiares campesinos generada por el grupo de investigación de donde se obtuvo el tiempo dedicado al cuidado, entre otras variables; así como la escala de carga de Zarit, para evaluar la carga de cuidador con una validez de criterio de $r=0,92$ ( $p<0,001$ en el ámbito internacional). En Colombia cuenta con una sensibilidad del $81,58 \%$, una especificidad del $96,35 \%$ con valores predictivos positivos del 75,61\% y negativos del 97,42\% (43). Dicho instrumento consta de 22 preguntas tipo Likert de 5 opciones (1-5 70 puntos), cuyos resultados se suman en un puntaje total (22-110 puntos). Este resultado clasifica al cuidador en "ausencia de sobrecarga" $(\leq 46)$, "so- 
brecarga ligera" (47-55) o "sobrecarga intensa" (56-110). Otro instrumento utilizado es el Índice de Barthel (IB), que mide el grado de dependencia del paciente en la realización de 10 actividades de la vida diaria: alimentarse, bañarse, vestirse, arreglarse, deambular, subir escaleras, trasladarse del sillón a la cama y viceversa, defecar, orinar y usar el sanitario, mediante la asignación de puntuaciones para cada actividad (0, 5, 10 y 15), que varían según la capacidad del paciente para realizarlas.

El grado de dependencia según la puntuación de la escala es el siguiente: de 0 a 20, dependencia total; de 21 a 60, dependencia severa; de 61 a 90, dependencia moderada; de 91 a 99, dependencia escasa, y 100, independencia. La confiabilidad del indice efectuada por Loewen y Anderson estableció una buena reproducibilidad interobservador con unos indices kappa de 0,47 a 1,0 e intraobservador de 0,84 a 0,97, respectivamente, y una consistencia interna con un índice de Cronbach de 0,86 y 0,92. Además, la confiabilidad obtenida del instrumento reportó un alfa de Cronbach de 0,93 (44).

\section{Análisis de los datos}

El almacenamiento y análisis de la información se efectuó con el software estadístico SPSS versión 17.0. Se practicaron análisis descriptivos de las variables. La información sociodemográfica de los cuidadores familiares se presenta en frecuencias. Se evaluó la relación de las variables utilizando valores asociados a las pruebas chi cuadrado $\left(\chi^{2}\right)$ con el fin de evaluar la existencia de asociaciones desde una perspectiva estadística; se consideró significativa la relación cuando el $p$ fue igual o menor a 0,05 con un nivel de confianza del $95 \%$.

\section{Consideraciones bioéticas}

Establecidas en la Resolución 8430 de 1993 del Ministerio de Salud de Colombia. Se obtuvo el aval del Comité de Ética de la Facultad de Enfermería de la Universidad Nacional, la autorización de la Alcaldía de Cáqueza y el consentimiento informado de los participantes. Los resultados obtenidos fueron presentados en la Casa de la Cultura del Municipio de Cáqueza a funcionarios y participantes del estudio.

\section{Resultados}

Los resultados se presentan en los siguientes aspectos: caracterización de los cuidadores familiares campesinos; carga del cuidador; grado de dependencia funcional de las personas ancianas; asociación entre la carga del cuidador y el grado de dependencia funcional de la persona anciana; asociación entre carga del cuidador y tiempo que lleva el cuidador campesino atendiendo a la persona anciana. 
En la tabla 1 se observa cómo la mayoría cuidadores familiares campesinos que participaron en el estudio son mujeres (76\%), la mitad de la muestra tiene 60 años y más, con bajo nivel económico $(100 \%)$ y de educación (90\%), predominan las casadas (40\%), son hijas los que cuidan a las personas ancianas con dependencia funcional (40\%) y que están a cargo de la persona anciana desde hace más de 8 años (64\%). Es de anotar que solo el 4\% lleva menos de un año. El 90\% de las familias se sostiene con aproximadamente medio salario mínimo (menos de 300.000 pesos o 150 dólares mensuales).

TABLA 1. Caracterización de los cuidadores familiares campesinos, Cáqueza, Cundinamarca

\begin{tabular}{|c|c|c|}
\hline $\begin{array}{l}\text { Caracteristicas sociodemográficas } \\
\text { de los cuidadores familiares campesinos }\end{array}$ & $\begin{array}{c}\text { General } \\
\text { Frecuencia }\end{array}$ & $\%$ \\
\hline $\begin{array}{l}\text { Género } \\
\text { Femenino } \\
\text { Masculino } \\
\text { Total }\end{array}$ & $\begin{array}{l}38 \\
12 \\
50 \\
\end{array}$ & $\begin{array}{r}76 \\
24 \\
100 \\
\end{array}$ \\
\hline $\begin{array}{l}\text { Edad } \\
19 \text { a } 34 \text { años } \\
35 \text { a } 59 \text { años } \\
60 \text { años o más } \\
\text { Total }\end{array}$ & $\begin{array}{l}4 \\
21 \\
25 \\
50\end{array}$ & $\begin{array}{r}8 \\
42 \\
50 \\
100\end{array}$ \\
\hline $\begin{array}{l}\text { Escolaridad } \\
\text { Sin escolaridad } \\
\text { Primaria } \\
\text { Secundaria incompleta } \\
\text { Total }\end{array}$ & $\begin{array}{c}6 \\
39 \\
5 \\
50\end{array}$ & $\begin{array}{r}12 \\
78 \\
10 \\
100\end{array}$ \\
\hline $\begin{array}{l}\text { Estado civil } \\
\text { Soltero(a) } \\
\text { Casado(a) } \\
\text { Viudo(a) } \\
\text { Divorciado(a) } \\
\text { Unión libre } \\
\text { Total } \\
\end{array}$ & $\begin{array}{c}15 \\
20 \\
3 \\
4 \\
8 \\
50 \\
\end{array}$ & $\begin{array}{r}30 \\
40 \\
6 \\
8 \\
16 \\
100\end{array}$ \\
\hline $\begin{array}{l}\text { Estrato socioeconómico } \\
\text { Uno } \\
\text { Dos } \\
\text { Total }\end{array}$ & $\begin{array}{c}47 \\
3 \\
50 \\
\end{array}$ & $\begin{array}{r}94 \\
6 \\
100 \\
\end{array}$ \\
\hline $\begin{array}{l}\text { Parentesco del cuidador con la persona cuidada } \\
\text { Esposo(a) } \\
\text { Hijo(a) } \\
\text { Nuera/yerno } \\
\text { Otro pariente } \\
\text { Total }\end{array}$ & $\begin{array}{c}11 \\
20 \\
1 \\
18 \\
50\end{array}$ & $\begin{array}{r}22 \\
40 \\
2 \\
36 \\
100\end{array}$ \\
\hline $\begin{array}{l}\text { Tiempo como cuidador } \\
1-3 \text { meses } \\
4-11 \text { meses } \\
1 \text { año } \\
2 \text { a } 3 \text { años } \\
4 \text { a } 8 \text { años } \\
\text { Más de } 8 \text { años } \\
\text { Total }\end{array}$ & $\begin{array}{c}1 \\
1 \\
3 \\
4 \\
9 \\
32 \\
50\end{array}$ & $\begin{array}{r}2 \\
2 \\
6 \\
8 \\
18 \\
64 \\
100\end{array}$ \\
\hline
\end{tabular}

Fuente: resultado según caracterización de los cuidadores familiares de personas ancianas, Cáqueza, Cundinamarca, 2011. 


\section{Carga del cuidador familiar}

Entre los cuidadores familiares campesinos predomina la sobrecarga intensa $(40 \%)$ y la sobrecarga ligera (16\%), influenciadas principalmente por la carga subjetiva, donde más de la mitad de los cuidadores siempre perciben miedo de lo que el futuro depare a su familiar (56\%) y creen que su familiar depende de ellos (56\%). Por la carga objetiva perciben que siempre su familiar parece esperar que ellos sean las personas que los cuiden (58\%), creen que no tienen suficiente dinero para cuidar a su familiar $(54 \%)$ y creen que podrian cuidar mejor de su familiar $(48 \%)$.

\section{Grado de dependencia de las personas ancianas}

Las personas ancianas presentan diferentes grados de dependencia funcional en las actividades de la vida diaria, según clasificación de Barthel: moderada (54\%), severa (14\%), total $(12 \%)$ y escasa $(20 \%)$, principalmente para realizar actividades como trasladarse entre sillas $(28 \%)$ y en la cama $(26 \%)$, en la dependencia total y las actividades para comer $(78 \%)$, el control de heces $(72 \%)$ y orina $(70 \%)$, el aseo personal, el bañarse y ducharse $(68 \%)$ en la dependencia severa.

\section{Asociación entre la carga del cuidador y el grado de dependencia funcional de la persona anciana}

Para determinar esta asociación se calculó $\chi^{2}$ con un nivel de confianza del $95 \%$. Se encontró un valor $\mathrm{p}=0,249$, lo que muestra que no existe asociación estadistica significativa entre la carga del cuidador familiar y el grado de dependencia funcional de la persona anciana campesina.

\section{Asociación entre la carga del cuidador y el tiempo como cuidador de la persona anciana}

Para determinar esta asociación se calculó $\mathrm{c}^{2}$ con un nivel de confianza del $95 \%$. Se halló un valor de $\mathrm{p}=0,476$, es decir no se encontró asociación estadistica significativa entre la carga del cuidador familiar y tiempo como cuidador, posiblemente porque las mujeres cuidadoras asumen el cuidado de su ser querido como un trabajo natural: "Solo hay que cuidarlo con amor, el tiempo es solo para eso" (45).

\section{Discusión}

Internacionalmente, "[...] la familia es el principal proveedor de asistencia al anciano dependiente" (46). Tal como afirma Wong, el apoyo familiar gana importancia relativa, sobre todo entre los grupos con bajos ingresos y que 
no cuentan con apoyo institucional. Las personas ancianas prefieren la compañía especialmente de un familiar, debido a que con la edad disminuyen las capacidades para hacer frente de manera independiente a las necesidades de la vida diaria (47).

En general, han sido poco exploradas las características de los cuidadores familiares en las zonas rurales $(30,45)$. Sin embargo, se encontró similitud con estudios donde la mayoría de las cuidadoras son mujeres (811,13); hijas o esposas de la persona dependiente del cuidado; con un nivel educativo bajo (12), con un gran número de horas dedicadas al cuidado $(12,48)$ y en tiempo de cuidado prolongado $(31,49)$. Así, el trabajo femenino del cuidado a los dependientes se entiende como una función natural transmitida de madre a hijas $(50,46)$ y las mujeres lo apropian de acuerdo con el grupo cultural al que pertenecen (51).

El papel de las mujeres como responsables del cuidado es visto como algo natural, que es transferido de generación en generación (14), especialmente en la familia campesina que se constituye en una unidad de producción y reproducción de sí misma; por lo tanto, subraya lazos de parentesco no solo con sus familiares por vínculos de consanguinidad, sino también con la vecindad, cuyo soporte, como afirma Galeski, citado en Mora y Sumpsi (52), son "todos esos factores que constituyen las especiales condiciones ambientales de las que la familia campesina, con sus funciones distintivas culturales, educativas y de 'seguridad social' deriva su existencia y durabilidad".

A pesar de que varios estudios han encontrado una asociación significativa entre el grado de dependencia funcional en las actividades básicas cotidianas de personas ancianas con presencia de carga para el cuidador $(\mathrm{p}<0,001)(27,48,53)$, son escasos los estudios que no hallan asociación entre estas variables (54). Por otra parte, no se localizaron suficientes estudios que reporten la asociación de carga de cuidado con tiempo de cuidado (12,31), y la mayoría han sido con el cuidado de personas con problemas mentales (31), lo que sugiere la importancia de continuar realizando estudios que aborden esta problemática, al igual que la asociación con el número de horas diarias $(12,48)$, dedicadas al cuidado de adultos mayores con dependencia funcional.

La no relación entre las variables carga del cuidador y el grado de dependencia funcional y el tiempo como cuidador de la persona anciana resulta un hallazgo llamativo para nuestro grupo que estudia la cultura, porque en la literatura internacional se reporta cómo las diferencias culturales pueden guardar relación con el bienestar físico y psicosocial del cuidador al ofrecer elementos resilientes a los cuidadores, debido a una evaluación positiva del cuidado, disponibilidad y el coping resultante de aspectos religiosos; sin embargo, se alerta cómo prácticas y actitudes culturales podrian coexistir con gran vulnerabilidad (15).

A partir de los hallazgos cualitativos de un estudio cualitativo previo con esta comunidad, se evidenció de qué modo el cuidado es ejercido por los cuidadores familiares campesinos como una responsabilidad, una retribución y no como una carga (45). Hallazgos similares se han encontrado con poblaciones rurales $(30,49)$. Estos nos motivan a continuar in- 
vestigando sobre los cuidadores familiares campesinos para establecer características del cuidado que ofrecen y la necesidad de sacar del escenario de lo privado este aporte social de los cuidadores familiares en el país para constituirlo objeto de políticas públicas.

En estudios del GCCS FE-UN $(36,45)$, el cuidado familiar a la persona anciana dependiente funcional, tanto en grupos rurales como urbanos, responde a valores y creencias de reciprocidad, obligación filial, afecto y deseo de ayudar. Se busca satisfacción por haber hecho lo que se debía y podía y así "no tener remordimientos". Igualmente, las creencias religiosas basadas en la consideración de una protección divina - un ser supremo que "da fuerza" para enfrentar las circunstancias adversas - y el cuidado al familiar pueden contribuir a mantener la esperanza, sentirse útil y amparado. Sin embargo, los cuidadores están dispuestos al sacrificio para aliviar a su familiar, soportan en silencio el dolor de la dependencia funcional y enfrentan un contexto de adversidad, las barreras institucionales y el escaso soporte institucional a su tarea (15).

En este sentido, es necesario crear servicios de apoyo formales para aliviar la carga de los cuidadores familiares, como programas específicos de intervención y servicios de respiro, que permitan al cuidador efectuar otras actividades (44) de acuerdo con su cultura y sistema familiar.

El estudio invita a revisar con atención el fenómeno de los cuidadores de minorías étnicas, quienes podrian tener menos carga por razones culturales. Es tiempo de aceptar el llamado a incluir los aspectos culturales en los estudios dirigidos a los cuidadores (15).

\section{Conclusiones}

El estudio no encontró asociación entre percepción de carga de cuidado en cuidadores familiares campesinos y dependencia funcional de personas ancianas, al igual que no encontró asociación entre percepción de carga del cuidador de cuidadores familiares y el tiempo de cuidado. La literatura muestra inconsistencias en los resultados de carga entre cuidadores familiares y su asociación con las variables del estudio y otras variables $(12,15,31)$.

Se requiere continuar con estudios que permitan establecer correlaciones con cuidadores campesinos específicos, por ejemplo, cuidadores campesinos de personas con trastornos mentales y con otras condiciones de salud, para reconocer asociaciones que afectan la percepción de la carga de cuidado. Así mismo, estudios de corte cualitativo que permitan explicaciones culturales al fenómeno como parte de la heterogeneidad de la experiencia y la construcción de significado frente al ofrecer el cuidado.

La mayoría de cuidadores familiares campesinos son personas ancianas que cuidan a otras personas ancianas y, por lo general, son hijas, mujeres, de bajos recursos económicos, casadas o solteras, con un nivel de escolaridad bajo y mucho tiempo empleado en las labores de cuidado que no son reconocidas socialmente. Sugiere la necesidad de politicas y programas dirigidos a las cuidadoras familiares campesinas para facilitar y hacer visible el cuidado que ofrecen (55). 
El estudio sugiere la necesidad de políticas públicas de cuidado, que favorezcan la participación de la mujer, combinando el trabajo productivo y el trabajo de cuidado, como lo realizan las cuidadoras familiares campesinas en el seno de las familias, donde se suministra cuidado a las personas dependientes, en un contexto de vulnerabilidad, sin un reconocimiento social ni económico.

Se requieren programas educativos dirigidos a las cuidadoras familiares campesinas mediante procesos de autorreconocimiento y autodeterminación.

Se presentaron algunas limitaciones en el estudio, como la dificultad en el acceso de los cuidadores a la cabecera municipal por razones económicas y por no encontrar quién los supla en su labor como cuidadores del anciano, de la casa y como productores familiares agropecuarios. Además, no se contó con el diagnóstico médico de la persona cuidada, sino que se tuvo como criterio de inclusión que presentará limitación física o sensorial no cognitiva o mental y dependiente de un cuidador familiar para las actividades de la vida diaria.

\section{Agradecimientos}

Las autoras agradecen a la Facultad de Enfermería de la Universidad Nacional de Colombia, a la Alcaldía de Cáqueza y a los cuidadores familiares campesinos de personas ancianas que participaron con sus valiosos aportes.

\section{Referencias}

1. Alpuche Ramírez V, Ramos del Río B, Rojas M, Figueroa López C. Validez de la entrevista de carga de Zarit en una muestra de cuidadores primarios informales. Psicología y Salud. 2008;18(2):237-45.

2. Barrero, C. García, S. Ojeda, A. Índice de Barthel (IB): un instrumento esencial para la evaluación funcional y la rehabilitación. Nuevos Horizontes. 2005;4(1-2).

3. Naciones Unidas. Una sociedad para todas las edades: Segunda Asamblea Mundial del Envejecimiento [internet]; Madrid 8 a 12 de abril de 2002 [citado 20 may 2011]. Disponible en: http://www. un.org/spanish/envejecimiento/newpresskit/hechos.pdf.

4. Comisión Económica para América Latina y el Caribe (Cepal), Naciones Unidas. Tendencias demográficas y protección social en América Latina y el Caribe [internet]. Santiago de Chile; 2008 [Citado 2011 mayo 21]. Disponible en: http://www.eclac.cl/publicaciones/ xml/5/33075/1c12864-P.pdf.

5. Arias S, Lozano A, López L, Velásquez V. La carga del cuidador: una revisión bibliográfica. Revista Kaana-uai "Cuidar de la vida". 2010;5(1):15-8.

6. Ballesteros Rodríguez J, Martín Carrasco M, Ibarra Gandiaga N, Loizaga Arnaiz C et al. Sobrecarga del cuidador de pacientes con enfermedad de Alzheimer y distrés psíquico: una asociación 
relegada en la valoración de las demencias. Actas Esp Psiquiatr. 2002;30:201-6.

7. Rogero García J. Los tiempos de cuidado: el impacto de la dependencia de los mayores en la vida cotidiana de sus cuidadores. Madrid: Ministerio de Sanidad y Política Social; 2010. p. 327-50.

8. Sánchez B. Habilidad de cuidado de los cuidadores de personas en situación de enfermedad crónica. En: El arte y la ciencia del cuidado. Bogotá: Unibiblos; 2002. p. 373-85.

9. Venegas B. Habilidad del cuidador y funcionalidad de la persona cuidada. Aquichan. 2006;6(1):137-47.

10. Zabala M et al. Características sociodemográficas de los cuidadores de ancianos. Documento procedente del Simposio Antropología de la Vejez; Chile; 2001.

11. Montalvo PA, Flórez TI. Características de los cuidadores de personas en situación de cronicidad. Cartagena (Colombia): un estudio comparativo. Revista Salud Uninorte [internet]. Barranquilla. 2008 [citado 20 mayo 2012]; 24(2). Disponible en: http://www.scielo.unal. edu.co/scielo.php.

12. Flores GE, Rivas RE, Seguel PF. Nivel de sobrecarga en el desempeño del rol del cuidador familiar de adulto mayor con dependencia severa. Revista Ciencia y Enfermería. 2012;XVIII(1):29-41.

13. Rose-Rego SK, Strauss ME, Smyth KA. Differences in the perceived well-being of wives and husbands caring for persons with Alzheimer's disease. Gerontologist. 1998;38(2):224-30.

14. Baptista BO, Beuter M, Girardon-Perlini NMO, Brondani CM, Budó MLD, Santos NO. A sobrecarga do familiar cuidador no âmbito domiciliar: uma revisão integrativa da literatura. Rev Gaúcha Enferm. 2012;33(1):147-56.

15. Pinquart M, Sörensen S. Spouses, adult children, and childrenin-law as caregivers of older adults: a meta-analytic comparison. Psychology and Aging. 2011;26(1):1-11.

16. Marim CM, Silva V, Taminato M, Barbosa DA. Effectiveness of educational programs on reducing the burden of caregivers of elderly individuals with dementia: a systematic review. Rev Latino-Am Enfermagem. 2013:267-75.

17. Oliveira DC, D'Elboux MJ. Estudos nacionais sobre cuidadores familiares de idosos: revisão integrativa. Rev Bras Enferm, Brasília. 2012 set-out;65(5):829-38.

18. Mason B, Harrison B. Telephone Interventions for family caregivers of patients with dementia. Alzheimer's Care Today. 2009;10(3):172-8.

19. Alfaro Ramírez O, Morales Vigil F, Vázquez Pineda S, Sánchez Román B, Ramos del Río U, Guevara L. Sobrecarga, ansiedad y depresión en cuidadores primarios de pacientes con dolor crónico y terminales. Rev Med Inst Mex Seguro Soc. 2008;46(5):485-94.

20. Martínez González L et al. Carga percibida del cuidador primario del paciente con parálisis cerebral infantil severa del Centro de Re- 
habilitación Infantil Teletón. Revista Mexicana de Medicina Física y Rehabilitación. 2008;20:23-9.

21. Nguyen M. Nurse's assessment of caregiver burden. Medsurg Nursing. 2009;18(3).

22. Giraldo C, Franco G, Correa L, Salazar M, Tamayo A. Cuidadores familiares de ancianos: quiénes son y cómo asumen este rol. Revista Facultad Nacional Salud Pública. 2005;23(2):7-15.

23. Barusch AS, Spaid WM. Spouse caregivers and the caregiving experience: Does cognitive impairment make a difference? Journal of Gerontological Social Work. 1996;25(3/4):93-106.

24. Crespo M, López J. Apoyo a cuidadores familiares mayores dependientes en el hogar: desarrollo del programa "Cómo mantener su bienestar". Madrid: Ministerio de Trabajo y Asuntos Sociales; 2007.

25. Thompson CW, Sugiyama T, Bell S et al. Landscape quality and quality of life [internet]; 2004. Disponible en: http://www.openspace.eca.ac.uk/conference2007/PDF/Summary_Paper__CWT__31_ Aug_07.AB_edit.pdf.

26. Thoits PA. Identity-relevant events and psychological symptoms: a cautionary tale. J Health Soc Behav. 1995;36(1):72-82.

27. Ocampo J, Herrera J, Torres P, Rodríguez J, Loboa L, García C. Sobrecarga asociada con el cuidado de ancianos dependientes. Colombia Médica. 2007;38(1).

28. Vargas L, Pinto N. Calidad de vida del cuidador familiar y dependencia del paciente con Alzheimer. Avances en Enfermería. 2010;XXVIII(1):116-28.

29. Carretero S, Garcés J, Ródenas F. La sobrecarga de las cuidadoras de personas dependientes: análisis y propuestas de intervención psicosocial [internet]. 2006. Disponible en http://www.imsersomayores. csic.es/documentos/documentos/polibienestar-sobrecarga-02.pdf.

30. Vestena J, Schwartz E, Manfrin R. Nursing's view of the care practices of rural families and the person with cáncer. Rev Esc Enferm USP. 2012;46(6):1372-9.

31. Lluch A, Morales A, Cabrera C, Betancourt M. Factores previsibles en la salud física y psicosocial del cuidador crucial del anciano con demencia en el hogar. Revista Cubana de Enfermería. 2010;26(2)3-13.

32. Cárdenas A, López L. Analysis matrix of resilience in the face of disability, old age and poverty. International Journal of Disability, Development and Education. 2010;57(2):175-89.

33. Cárdenas A, López L. Resiliencia en la vejez. Rev Salud Pública. 2011;13(3):528-40.

34. Castellanos F, López L. Tejiendo explicaciones sobre vejez, discapacidad y pobreza en los cerros nororientales de Bogotá, Colombia. Investig Enferm. Imagen Desarr. 2011;13(2):27-47.

35. López L, Cataño N, López H, Velásquez V. Diversidad cultural de sanadores tradicionales afrocolombianos: preservación y conciliación de saberes. Aquichan. 2011;3:287-304. 
36. Mejía L, López L. La familia y cultura: una conexión innovadora para el cuidado de la salud. Index de Enfermería. 2010;19(2-3):13843.

37. Velásquez V, López L, López H, Cataño N, Muñoz E. Efecto de un programa educativo para cuidadores de personas ancianas: una perspectiva cultural. Rev Salud Pública. 2011;13(3):468-569.

38. Velásquez V, López L, López H, Cataño N. Tejido de significados en la adversidad: discapacidad, pobreza y vejez. Hacia la Promoción de la Salud. 2011;16(2):121-31.

39. Castellanos F, López L. Mirando pasar la vida desde la ventana: significados de la vejez y la discapacidad de un grupo de ancianos en un contexto de pobreza. Investig Enferm. Imagen Desarr. 2010;12(2):37-53.

40. Forero Álvarez J. Economía campesina y sistema alimentario en Colombia: aportes para la discusión de la seguridad alimentaria [internet]. Bogotá; 2003 [citado feb 2014]. Disponible en: http://www. javeriana.edu.co/ear/d_des_rur/documents/campesinadoysistemaalimentarioencolombia.pdf.

41. Food and Agriculture Organization (FAO). Participación campesina para una agricultura sostenible en países de América Latina [internet]; 1994 [citado feb 5 2014]. Disponible en: http://www.fao.org/ docrep/003/t3666s/t3666s04.htm.

42. Naciones Unidas. Declaración sobre los derechos de los campesinos y de otras personas que trabajan en las zonas rurales [internet]; 2013 [citado 10 mar 2014]. Disponible en: http://www.ohchr.org/Documents/HRBodies/HRCouncil/WGPleasants/A-HRC-WG-15-1-2_ sp.pdf.

43. Vélez Lopera JM, Berbesí Fernández D, Cardona Arango D, Ordóñez Molina J. Validación de escalas abreviadas de Zarit para la medición de sindrome del cuidador primario del adulto mayor en Medellín. Aten Primaria. 2012;44(7):411-6.

44. Carretero Gómez S, Garcés Ferrer J, Ródenas Rigla F. La sobrecarga de las cuidadoras de personas dependientes: análisis y propuestas de intervención psicosocial [internet]. Disponible en: http://www. insersomayores.csic.es/documentos/documentos/polibienestar-sobrecarga-02.pdf.

45. Bejarano HTA. Cuidados populares brindados por cuidadores familiares a personas ancianas en situación de discapacidad y pobreza, área rural de Cáqueza. Tesis de maestría, Universidad Nacional de Colombia; 2012.

46. Montoro J. Las consecuencias psicosociales del cuidado informal a personas mayores. Rev Int Sociol. 1999;(23):7-29.

47. Guzmán J, Huenchuan S. Politicas hacia las familias con personas mayores: el desafío del derecho al cuidado en la edad avanzada. En: Naciones Unidas, Cepal. Políticas hacia las familias, protección e inclusión sociales: seminarios y conferencia [internet]. Chile; 2005. 
Disponible en: http://www.bidihmujer.salud.gob.mx/documentos/7/ Politicas \%20hacia \%201as \%20familias.pdf.

48. Pereira R, Barros dos Santos E, Silva J, Marques S, Partezani A. Sobrecarga dos cuidadodres de idosos com acidente vascular cerebral. Rev Esc Enferm USP. 2013;47(1):185-92.

49. Vidal Gutiérrez D, Zavala Gutiérrez M, Castro Salas M, Quiroga López P, Klaasen Pinto G. El significado del paciente con demencia para el cuidador, en una comunidad urbana y rural. Revista de Servicio Social. 1998;1(2).

50. Giraldo CI, Franco GM, Correa LS, Salazar MO, Tamayo AM. Cuidadores familiares de ancianos: quiénes son y cómo asumen este rol. Revista de la Facultad Nacional de Salud Pública. 2005;23(2):7-15.

51. Giraldo Molina CI, Franco Agudelo GM. Calidad de vida de los cuidadores familiares: Aquichán. 2006;6(1):38-53.

52. Mora J, Sumpsi J. Desarrollo rural: nuevos enfoques y perspectivas [internet]. Proyecto Regional de Cooperación Técnica para la Formación en Economía y Politicas Agrarias y de Desarrollo Rural en América Latina; 2004. Disponible en: http://190.11.224.74:8080/ jspui/bitstream/123456789/577/1/SumpsiyMora.pdf.

53. Di Mattei VE, Prunas A, Novella L, Marcone A, Cappa SF, Sarno L. The burden of distress in caregivers of elderly demented patients and its relationship with coping strategies. Neurol Sci. 2008;29(6):383-9.

54. Zarit SH, Rever KE, Bach-Peterson J. Relatives of the impaired elderly: Correlates of feelings of burden. Gerontologist. 1980;20:64955.

55. Rico MN, Maldonado VC. ¿Qué muestra la evolución de los hogares sobre la evolución de las familias en América Latina? En: Rico M, Maldonado C, editores. Las familias latinoamericanas interrogadas: hacia la articulación del diagnóstico, la legislación y las políticas. Santiago: Naciones Unidas; 2011 [citado 12 feb 2014]. Disponible en: http://www.eclac.org/publicaciones/xml/6/42586/ssc-61-familiaslatinoamericanas-feb-2011. 